Contributors: CPG was principal investigator and is guarantor. He designed the study along with ARG and HMK. Study data were abstracted by CPG and ARG. All authors collaborated on interpretation of the data and writing the manuscript.

Funding: No external funding.

Competing interests: None declared.

Blumenthal D. Ethics issues in academic-industry relationships in the life sciences: the continuing debate. Acad Med 1996;71:1291-6.
2 Bodenheimer T. Uneasy alliance-clinical investigators and the pharmaceutical industry. N Engl J Med 2000;342:1621-6.

3 Uniform requirements for manuscripts submitted to biomedical journals: International Committee of Medical Journal Editors. Ann Intern Med 1997;126:36-47.

4 Hussain A, Smith R. Declaring financial competing interests: survey of five general medical journals. BMJ 2001;323:263-4.

5 Davidoff F, DeAngelis C, Drazen JM, Hoey J, Hojharrd L, Horton R Sponsorship, authorship, and accountability. Lancet 2001; 358:854-6.

(Accepted 14 December 2002)

\title{
Total and cause specific mortality among Swedish women with cosmetic breast implants: prospective study
}

\author{
V C M Koot, P H M Peeters, F Granath, D E Grobbee, O Nyren
}

The potential health hazards of breast implants have been heavily debated for the past decade, yet only one study has reported on long term mortality among women with such implants, and around one fifth of the participants were lost to follow up. ${ }^{12}$ We assessed total and cause specific mortality among Swedish women who underwent augmentation mammoplasty between 1965 and 1993. As a desire for cosmetic surgery represents underlying psychopathology in some patients, we hypothesised that deaths due to suicide may be over-represented. ${ }^{3}$

\section{Subjects and methods}

Details about accrual of the cohort have been given elsewhere. ${ }^{4}$ We obtained records from the Swedish Inpatient Register of all 15-69 year old women who had had breast implants $(n=7585)$ in 1965-93. We identified records with erroneous registration numbers or where emigration or death occurred before surgery through linkages with registers held by Statistics Sweden, using the unique national registration numbers. We excluded such records and records where surgery occurred at hospitals without surgical services $(\mathrm{n}=138)$. We also excluded women who had received an implant after surgery for breast cancer $(n=3926)$, identified through the cancer register. The final study cohort comprised 3521 women, with a mean age of 31.6 (SD 8.6) years.

Follow up started on the day of first implantation surgery and stopped at date of emigration, death, or end of follow up (31 December 1994), whichever occurred first. The cohort members were followed for an average of 11.3 (range 0.3-29.9) years, corresponding to 39735 person years at risk. We compared the observed number of deaths with the expected number of deaths, the ratio of these two numbers giving the standardised mortality ratio. We obtained the expected number of deaths by multiplying the observed number of person years at risk in the cohort, divided into 5 year age strata and 1 calendar year strata, by the stratum specific mortality rates, derived from official Swedish death statistics. The standardised mortality ratio can therefore be viewed as a measure of relative risk, with the Swedish female population matched for age and calendar year serving as reference. We calculated 95\% confidence intervals, assuming that the number of observed events followed a Poisson distribution. We coded underlying causes of death according to the international classification of diseases ( 7 th, 8 th, and 9th revisions) into suicide, unintentional injury, cardiovascular diseases, malignancies, and other causes.

\section{Results}

Although 58.7 deaths were expected, 85 women died (standardised mortality ratio $1.5,1.2$ to 1.8 ; table). Fifteen women committed suicide, compared with 5.2 expected deaths $(2.9,1.6$ to 4.8$)$. Excess deaths were also due to malignant disease $(1.4,1.0$ to 1.9$)$, mainly lung cancer. The number of deaths for all other causes was close to expected.

\section{Comment}

Women who undergo cosmetic surgery for breast augmentation are more likely to commit suicide than women from the general population. The $50 \%$ excess mortality found by us in our prospective study of 3000 Swedish women contrasts with the decreased mortality reported from the United States. ${ }^{2}$ This may reflect different reasons for self selection for plastic surgery or may be an effect of losses to follow up in the American study. Both the American study and our study did, however, show an increased risk for suicide in women opting for breast augmentation. Our excess

Standardised mortality ratios for total and cause specific mortality in 3521 Swedish women with cosmetic breast implants

\begin{tabular}{lccc} 
Cause of death & $\begin{array}{c}\text { Observed } \\
\text { No of deaths }\end{array}$ & $\begin{array}{c}\text { Expected } \\
\text { No of deaths* }\end{array}$ & $\begin{array}{c}\text { Standardised mortality ratio } \\
\text { (95\% Cl) }\end{array}$ \\
\hline All causes & 85 & 58.7 & $1.5(1.2$ to 1.8$)$ \\
\hline Suicide & 15 & 5.2 & $2.9(1.6$ to 4.8$)$ \\
\hline Unintentional injury & 10 & 5.6 & $1.8(0.9$ to 3.3$)$ \\
\hline Cardiovascular disease & 11 & 11.2 & $1.0(0.5$ to 1.8$)$ \\
\hline Malignancies: & 36 & 25.9 & $1.4(1.0$ to 1.9$)$ \\
\hline Lung & 8 & 2.7 & $3.0(1.3$ to 5.9$)$ \\
\hline Cervix uteri & 3 & 1.2 & $2.5(0.5$ to 7.4$)$ \\
\hline Breast & 4 & 6.7 & $0.6(0.2$ to 1.6$)$ \\
\hline Other causes & 13 & 10.8 & $1.2(0.6$ to 2.1)
\end{tabular}

${ }^{*}$ Based on age specific and calendar year specific death rates in total female Swedish population.
Julius Centre for Health Sciences and Primary Care, University Medical Centre, Box 85500 3508 GA Utrecht,

Netherlands

V C M Koot clinician

P H M Peeters associate professor D E Grobbee professor

Department of Medical

Epidemiology, Karolinska Institute, Box 281, S-171 77

Stockholm, Sweden

F Granath associate professor

O Nyren professor

Correspondence to: V C M Kuck-Koot, Comprehensive

Cancer Centre

Middle

Netherlands, PO Box 19079, 3501 DB Utrecht,

Netherlands kuckkoot@ikmn.nl

BMJ 2003;326:527-8 
mortality was explained by the excess of suicides and deaths from malignant disease. Deaths due to malignancy were mainly linked to smoking, previously shown as common in our cohort. ${ }^{5}$ Given the well documented link between psychiatric disorders and a desire for cosmetic surgery, the increased risk for death from suicide may reflect a greater prevalence of psychopathology rather than a causal association between implant surgery and suicide. ${ }^{3}$ Surgeons evaluating candidates for breast implant surgery need to be vigilant for subtle signs of psychiatric problems.

Contributors: VCMK, the principal investigator, discussed the core ideas, performed the record linkages, outlined and performed analyses, and wrote most of the paper. PHMP discussed the core ideas and participated in data interpretation and writing of the paper. FG participated in discussions about the core ideas, made suggestions about analyses, and helped VCMK with the practical analysis. DEG discussed the core ideas, the design of the study, the interpretation of the data, and writing of the paper. ON initiated the research, discussed the core ideas, formulated the primary study hypothesis, made suggestions about analyses and interpretation of the data, and supervised the writing. DEG and ON will act as guarantors for the paper.

Funding: This work was supported by a contract from the International Epidemiology Institute, Rockville, MA. VCMK was financially supported by the Netherlands Organization for Scientific Research.

Competing interests: None declared.

Angell M. Evaluating the health risks of breast implants: the interplay of medical science, the law, and public opinion. $N$ Engl J Med 1996;334:1513-8.

2 Brinton LA, Lubin JH, Burich MC, Colton T, Hoover RN. Mortality among augmentation mammoplasty patients. Epidemiology 2001;12:321-6.

3 Hasan JS. Psychological issues in cosmetic surgery: a functional overview. Ann Plast Surg 2000;44:89-96.

4 Nyren O, Yin L, Josefsson S, McLaughlin JK, Blot WJ, Engqvist M, et al. Risk of connective tissue disease and related disorders among women with breast implants: a nation-wide retrospective cohort study in Sweden. BMJ 1998;316:417-22.

5 Fryzek JP, Weiderpass E, Signorello LB, Hakelius L, Lipworth L, Blot WJ, et al. Characteristics of women with cosmetic breast augmentation surgery compared with breast reduction surgery patients and women in the general population of Sweden. Ann Plast Surg 2000;45:349-56.

(Accepted 17 January 2003)

\title{
Systematic review of lipid lowering for primary prevention of coronary heart disease in diabetes
}

\author{
Apoor S Gami, Victor M Montori, Patricia J Erwin, Mehmood A Khan, Steven A Smith for the \\ Evidence in Diabetes Enquiry System (EVIDENS) Research Group
}

Department of Internal Medicine, Mayo Clinic, 200 First Street SW, Rochester MN 55905, USA

Apoor S Gami instructor in medicine

Division of Endocrinology, Diabetes,

Metabolism,

Nutrition, and

Internal Medicine,

Mayo Clinic

Victor M Montori assistant professor

Mehmood A Khan associate professor

Steven A Smith associate professor

Mayo Medical

Libraries, Mayo

Clinic

Patricia J Erwin

head reference

librarian

Correspondence to:

S A Smith

smith.steven@mayo.edu

BMJ 2003;326:528-9

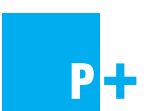

The studies included in the review are listed on bmj.com
Patients with diabetes are at high risk of coronary heart disease. Leading organisations have recommended that all diabetic patients should be treated as aggressively as patients with established coronary heart disease. ${ }^{1}$ Randomised trials have shown the efficacy of reducing low density lipoprotein concentrations in patients without coronary heart disease. Large trials and meta-analyses of such trials would be expected to provide information on diabetic patients. We therefore systematically examined the available data on lowering low density lipoprotein concentrations in diabetic patients without coronary heart disease.

\section{Methods and results}

The review protocol is available from the authors. We searched Medline and eight other electronic databases (including five clinical trials databases) and proceedings from pertinent scientific meetings. We attempted to contact the authors of trials reporting incomplete data but received no responses. We reviewed the bibliographies of all retrieved publications.

Eligible trials randomised patients to lipid lowering interventions; included patients without coronary heart disease; and measured myocardial infarction, death from coronary heart disease, or all cause mortality. Eligible meta-analyses pooled data from similar trials. We excluded studies available only as abstracts. There were no language exclusions. We also included the Medical Research Council/British Heart Foundation heart protection study, which was pub- lished after our search. ${ }^{2}$ A list of included trials and meta-analyses is available on bmj.com

The 14 eligible trials randomised 132977 patients without coronary heart disease, and diabetes status was stated for 1799 patients (1.3\%). Three trials provided clinical endpoints for 454 diabetic patients. In addition, the heart protection study randomised 3982 diabetic patients without coronary heart disease (table).

We found 13 meta-analyses that included up to 38 trials and 146854 patients. None presented data for diabetes subgroups. One meta-analysis postulated that diabetes might account for differences between trials, but incomplete reporting in the trials limited the analysis.

\section{Comment}

Inclusion and reporting biases in randomised controlled trials and meta-analyses limited our assessment of the efficacy of lowering low density lipoprotein concentration in diabetic patients without coronary heart disease. Most trials of lipid lowering interventions for primary prevention of coronary heart disease excluded diabetic patients by varied and ambiguous criteria. Consequently, these trials included few patients with diabetes. Those who were included were poorly characterised in terms of type and duration of diabetes, severity of complications, and metabolic control. It is therefore unclear whether the diabetes subgroups represent the general diabetic population. 